\title{
Regular Heel-raise Training Focused on the Soleus for the Elderly: Evaluation of Muscle Thickness by Ultrasound
}

\author{
Katsuo Fujiwara ${ }^{1)}$, Hiroshi Toyama ${ }^{1)}$, Hitoshi Asai ${ }^{2)}$, Kaoru Maeda $^{3)}$ and Chie Yaguchi ${ }^{1)}$ \\ 1) Department of Human Movement and Health, Graduate School of Medical Science, Kanazawa University \\ 2) Department of Physical Therapy, Graduate School of Medical Science, Kanazawa University \\ 3) Department of Physical Therapy, Faculty of Health Sciences, Morinomiya University of Health Sciences
}

\begin{abstract}
The soleus, one of the triceps surae muscles, greatly contributes to standing and walking. Strength training focused on the soleus could be important to prevent agerelated deterioration in these functions. We therefore investigated the effects of regular heel-raise training focused on the soleus for the elderly. Forty-nine healthy women aged 60 to 79 years trained for at least 40 days in a period of two months. Training consisted of a set of 100 repetitions per day of heel-raise with both legs in a standing position. The training effect was evaluated by changes in each muscle thickness of the soleus and gastrocnemius medialis, which was measured using an ultrasound scanner, as well as plantar flexor strength. The subjects' ability to perform the training and their subjective opinions of its effects were assessed by a questionnaire survey. Plantar flexor strength and thicknesses of the soleus and gastrocnemius medialis were increased significantly by the training. The percentage increase in thickness was significantly greater for the soleus than for the gastrocnemius medialis ( $12.7 \%$ vs. $6.6 \%)$. These improvements did not significantly correlate with age. The questionnaire results suggested that the elderly were able to safely and easily perform the heel-raise training at home. This study demonstrated that regular heel-raise training is an effective muscle training method for the elderly, focused on the soleus. J Physiol Anthropol 29(1): 23-28, 2010 http:// www.jstage.jst.go.jp/browse/jpa2
\end{abstract}

[DOI: $10.2114 / \mathrm{jpa} 2.29 .23]$

Keywords: muscle training, soleus, gastrocnemius, plantar flexor strength, the elderly

\section{Introduction}

Among the postural muscles, the triceps surae shows the greatest activation during a quiet standing posture, which is a basic posture for humans (Okada, 1971). In addition, this muscle is strongly activated in the stance phase of walking to maintain the standing posture and generate forces for propulsion (Inman et al., 1981). During walking, the triceps surae generates $93 \%$ of plantar flexion torque (Haxton, 1944), and the soleus (SoL) greatly contributes to this torque (Perry, 1992). The triceps surae accounts for $73 \%$ of the total muscle volume of the plantar flexors, and SoL accounts for $41 \%$ (Fukunaga et al., 1996). Deterioration in triceps surae strength reportedly affects postural steadiness (Fujiwara et al., 1982) and the ability to walk (Nadeau et al., 1999). Strength training of the triceps surae could therefore be important to prevent age-related deterioration in these functions. Studies on strength training of the triceps surae have demonstrated an improvement in plantar flexor strength (PFS) and in triceps surae muscle volume (Ferri et al., 2003; Morse et al., 2005).

In terms of histology, SoL and gastrocnemius (GM) differ in their proportion of slow- and fast-twitch muscle fibers: the proportion of slow-twitch muscle fibers is approximately $90 \%$ in SoL and 50\% in GM (Johnson et al., 1973; Trappe et al., 2001). Slow-twitch muscle fibers are recruited at a lower muscle load compared with fast-twitch muscle fibers (Henneman et al., 1965). This indicates that the suitable training intensity differs between these two types of muscle fibers. It is reported that low-intensity muscle strength training causes significant hypertrophy of slow-twitch muscle fibers (Shono et al., 2002). The relative muscle load of triceps surae while maintaining a raised heel position is 5 to $40 \%$ of maximum voluntary contraction (Okada, 1971). Maximum repetition of the muscle contraction is reportedly 60-110 times at $30 \%$ of maximum voluntary contraction (Ikai et al., 1965; Berger and Hardage, 1967). In addition, muscle blood flow is maintained or increased in such muscle load (Humphreys and Lind, 1963; Wernbom et al., 2006). Therefore, it is likely that such low muscle load is suitable for training of slow-twitch muscle fibers, the contraction of which is strongly dependent on muscle blood flow (Bonde-Petersen and Robertson, 1981; Petrofsky et al., 1981). Hence, the heel-raise training with approximately 100 repetitions should focus on slow-twitch muscle fibers in the triceps surae. It is presumed that this 
training would be especially effective for SoL, which has a high proportion of slow-twitch muscle fibers. Therefore, it is important to separately evaluate the training effect in GM and SoL.

Muscle strength is proportional to muscle cross-sectional area (Ikai and Fukunaga, 1968), and muscle thickness is an important factor related to muscle cross-sectional area. Many researchers have used ultrasound to measure muscle thickness (Reimers et al., 1996; Narici and Cerretelli, 1998), and this is noninvasive and straightforward to assess the muscle thickness of SoL and GM. Because ultrasound apparatus is small and lightweight, it can be used to measure muscle thickness on a large population outside the laboratory.

Muscle hypertrophy has been observed from 5 to 9 weeks after onset of muscle training (Young et al., 1983; Ploutz et al., 1994). In order for the elderly to continue muscle training over a long period, the training method must be safe, easy, and able to be performed frequently.

Therefore, in the present study, elderly participants performed daily heel-raise training focused on SoL for two months. Before and after the training period, PFS and muscle thicknesses of SoL and gastrocnemius medialis (GMM) were measured. Our purpose was to examine the hypothesis that this training would be effective in strengthening triceps surae, and that the effect would be more noticeable in SoL than in GMM.

\section{Methods}

\section{Participants}

Participants were 49 healthy female volunteers, ranging in age from 60 to 79 years $($ mean $=69.0, \mathrm{SD}=5.0)$, who had not performed any regular training or exercise. Their mean height and weight before training were $147.7(\mathrm{SD}=6.3) \mathrm{cm}$ and 51.9 $(\mathrm{SD}=7.1) \mathrm{kg}$, respectively. No significant differences in height and weight were found between before and after training. We recruited participants from community centers in Suzu and Kanazawa Cities, Ishikawa Prefecture, Japan, and explained the purpose and method of this study. Participants understood this information and agreed to voluntarily participate in this study. All participants provided informed consent to participate in the study after being provided with an explanation of the experimental protocol, which had been approved by the institutional ethics committee of Kanazawa University. The health status of participants was preliminarily assessed by a short questionnaire, and only those without limitation of daily activities due to cardiopulmonary, musculoskeletal, or neurological disease, and those with no pain or no analgesic use were enrolled.

\section{Training protocol}

For the heel-raise training, participants simultaneously lifted both heels from a standing position to bear their weight on their forefeet. Participants were instructed to lift the heels slowly at intervals of about $2 \mathrm{~s}$ and to maintain the heel-raise position for about $1 \mathrm{~s}$ each time, in order to avoid a sudden high load on the triceps surae. The heel-raise position was set at the height at which participants were able to repeat the training 100 times. Training consisted of a set of 100 repetitions per day for at least 40 days in the period of two months (62 days). The actual mean training period was 59.5 $(\mathrm{SD}=3.8)$ days.

\section{Measurement of PFS}

The maximal isometric muscle strength of plantar flexor was measured in a sitting position using an instrument to measure PFS invented by Fujiwara (Fujiwara et al., 1988) (Fig. 1). The right knee and ankle were kept in $90^{\circ}$ flexion, and the knee was secured with a belt at the top of the leg. PFS was measured as the force exerted at the metatarsal heads of the right foot. To avoid the measurement being affected by recoil motion in other regions of the body, when they generated the plantar flexion force, participants were instructed not to touch the floor with their left foot, and not to rotate the pelvis or trunk. In addition, they were asked to generate the plantar flexion force during expiration to prevent rapid elevation of blood pressure. Muscle strength measurement was performed twice and the higher value was used for calculations.

\section{Measurement of muscle thicknesses of SoL and GMM}

The thicknesses of SoL and GMM were measured using a real-time B-mode ultrasound scanner (Hitachi Medico, EUB405B, Japan) with a $3.8-\mathrm{cm}, 10-\mathrm{MHz}$ linear array probe. Measurements were performed directly on the screen using electronic calipers with $0.1-\mathrm{mm}$ resolution. To measure the right side, participants sat on a chair with the right foot on the floor, and the target point was defined as follows. First, the level of maximum girth of the right leg was determined using a tape measure. Next, at this level, the midpoint of the mediolateral width of GMM was marked with a red permanent marker as the target point. During ultrasound measurement, the longitudinal axis of the right leg was parallel to the vertical axis of gravity, with the ankle at zero degrees of dorsiflexion/

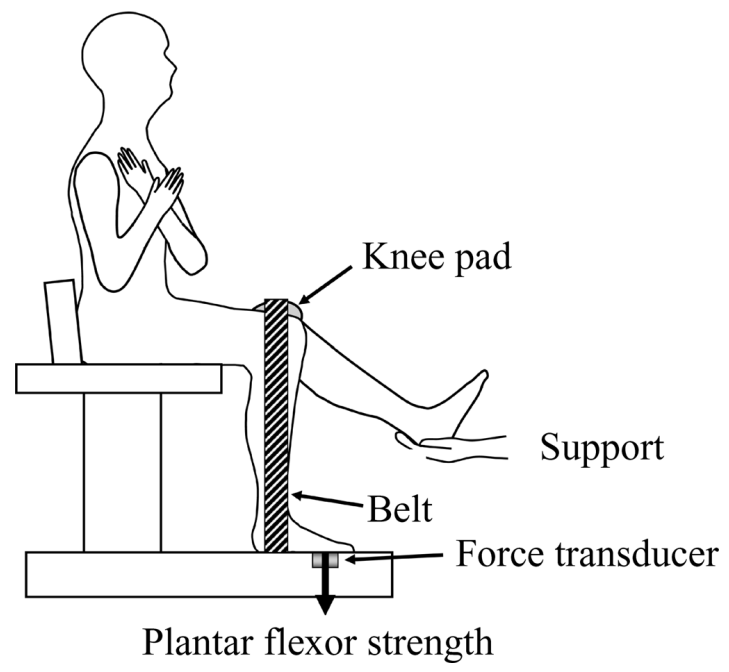

Fig. 1 Measurement of plantar flexor strength. 
plantar flexion. For ultrasound scanning, the head of the ultrasound probe was coated with coupling gel and applied to the target point. The probe was oriented in the axial plane, perpendicular to the muscle. The ultrasound image under the probe was displayed on a computer screen. We measured the thickness of SoL and GMM under the target point. During scanning, great care was taken to manipulate the probe so that the fasciae were parallel and to avoid compressing the dermal surface. GMM thickness was defined as the anterior-posterior distance between the midpoints of the fascia posterior to the muscle and the fascia separating GMM from SoL. SoL thickness was defined as the anterior-posterior distance between the midpoints of the fascia separating GMM from SoL and the fascia anterior to SoL (Fig. 2). The reliability and the validity of ultrasound measurement of muscle thickness were examined in our previous study (Fujiwara et al., 2010).

Questionnaire on the implementation of training and the subjective assessment of training effects

Using a questionnaire, participants were asked to report training place and time, occurrence of training-associated injuries or problems, the difficulty of training, and their subjective assessment of training effects and the latency of these effects. For questions of training place, time, injuries, problems, and training effect, multiple answers were allowed.

\section{Statistical analyses}

A paired $t$ test was used to examine statistical differences between data collected pre- and post-training. A Student's $t$ test or Welch's $t$ test was used to assess the difference in muscle thickness increase between both muscles depending on
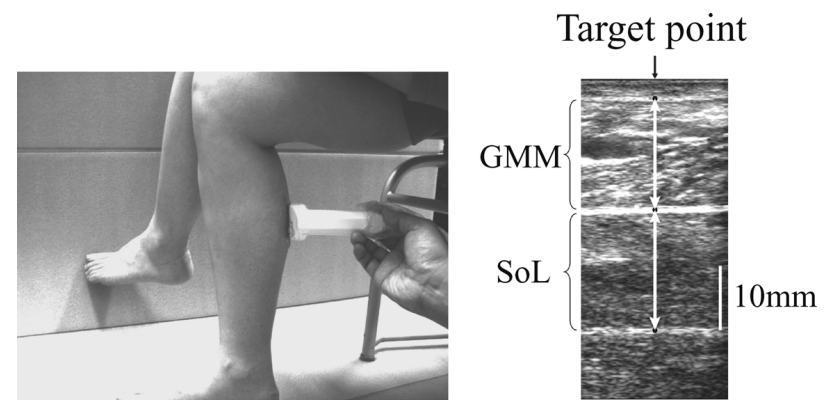

Fig. 2 The experimental set-up for measurement of the muscle thickness of gastrocnemius medialis (GMM) and soleus (SoL). whether a significant difference in variance was observed or not. Pearson correlation was calculated to assess the relationship between measurements. The significance of correlation coefficients was evaluated with the $\mathrm{Z}$ test after performing Fisher's $Z$ transformation. All statistical analyses were performed using SPSS 14.0J (SPSS, Japan). The alpha level was set at $p<0.05$.

\section{Results}

Age-related change in PFS and muscle thickness

Before training, a significant negative correlation was found between age and PFS $(r=-0.54, p<0.01)$ and between age and GMM thickness $(r=-0.44, p<0.01)$, indicating that both PFS and GMM thickness decreased with age. On the other hand, no significant correlation was found between age and SoL thickness $(r=-0.28, n s)$.

\section{Training effects on PFS and muscle thickness (Table 1)}

PFS after training was significantly larger than that before training $(727.7 \mathrm{~N}$ vs. $589.4 \mathrm{~N}, t=12.34, p<0.001)$; this percentage increase was $25.8 \%(\mathrm{SD}=17.0)$. SoL and GMM were significantly thicker after training than before it (SoL: $19.8 \mathrm{~mm}$ vs. $17.7 \mathrm{~mm}, t=7.02, p<0.001$; GMM: $16.2 \mathrm{~mm}$ vs. $15.3 \mathrm{~mm}, t=3.73, p<0.001)$. The percentage increase in thickness was significantly larger for SoL than for GMM $(12.7 \%$ vs. $6.6 \%, t=2.45, p<0.05)$. The relative thickness of GMM to SoL after training was significantly smaller than that before training $(0.85$ vs. $0.90, t=2.81, p<0.01)$.

\section{Relationships between each measurement before training and the percentage increase in PFS and muscle thickness}

Figure 3 shows the relationship between each measurement before training and its percentage increase. PFS and SoL thickness increased with training in all participants. Significant negative correlations were found between PFS before training and its percentage increase $(r=-0.51, p<0.001)$, and between SoL thickness before training and its percentage increase $(r=-0.34, p<0.05)$. GMM thickness did not increase in 12 of the 49 participants; these individuals had a relatively high thickness before training. A significant negative correlation was found between GMM thickness before training and its percentage increase $(r=-0.59, p<0.001)$.

Figure 4 shows the relationship between the relative

Table 1 Training effects on plantar flexor strength and muscle thickness

\begin{tabular}{|c|c|c|c|c|c|c|}
\hline & \multicolumn{2}{|c|}{ before training } & \multicolumn{2}{|c|}{ after training } & \multicolumn{2}{|c|}{ Percentage increase } \\
\hline & mean & S.D. & mean & S.D. & mean & S.D. \\
\hline Plantar flexor strength & $589.4 \mathrm{~N}$ & 164.8 & $727.7 \mathrm{~N}^{* * *}$ & 171.6 & $25.8 \%$ & 17.0 \\
\hline \multicolumn{7}{|l|}{ Muscle thickness } \\
\hline Soleus & $17.7 \mathrm{~mm}$ & 3.6 & $19.8 \mathrm{~mm}^{* * *}$ & 3.9 & $12.7 \%$ & 13.3 \\
\hline Gastrocnemius medialis & $15.3 \mathrm{~mm}$ & 3.3 & $16.2 \mathrm{~mm}^{* * *}$ & 2.9 & $6.6 \%$ & 11.1 \\
\hline Relative thickness & 0.90 & 0.22 & $0.85^{* *}$ & 0.20 & - & - \\
\hline
\end{tabular}




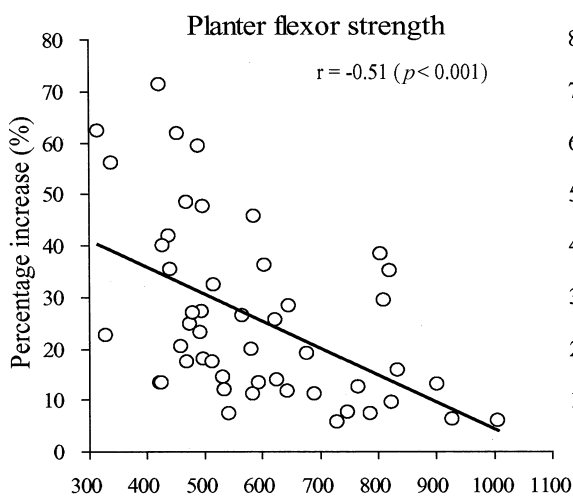

Planter flexor strength $(\mathrm{N})$ before training

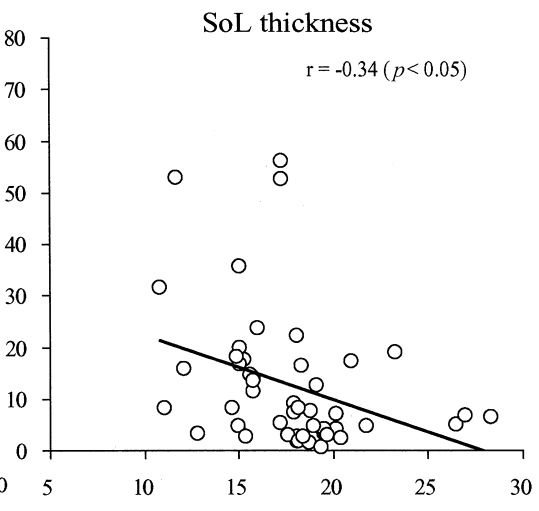

SoL thickness $(\mathrm{mm})$ before training

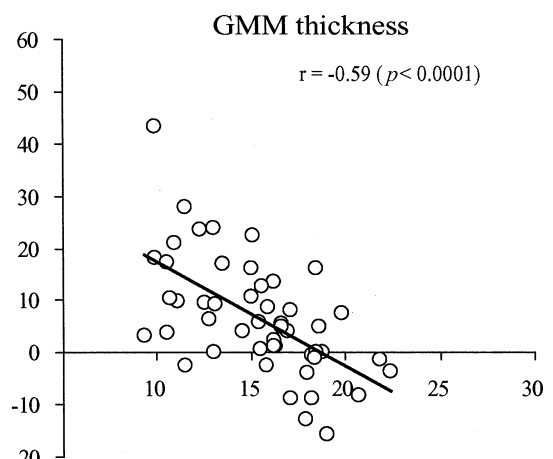

GMM thickness (mm) before training

Fig. 3 Relationships between each measurement before the training and percentage increase in plantar flexor strength and muscle thickness.
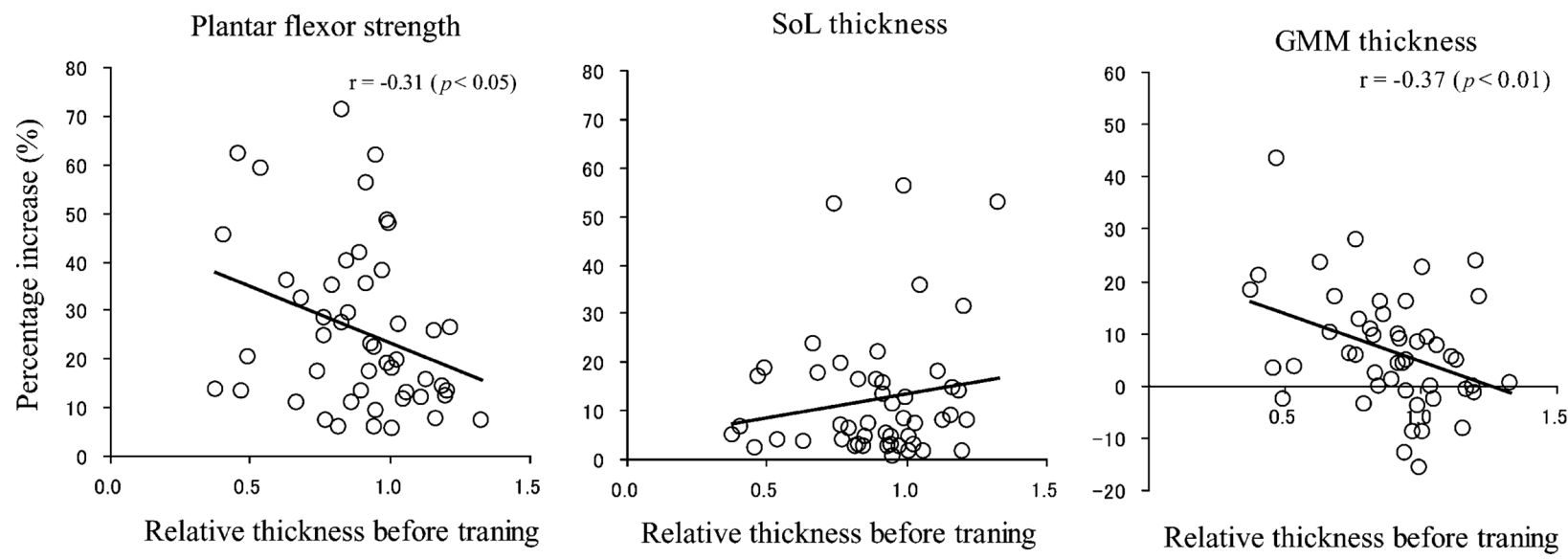

Fig. 4 Relationships between relative thickness of GMM to SoL before the training and percentage increase in plantar flexor strength and muscle thickness.

thickness of GMM to SoL before training and the percentage increase in each measurement. A significant negative correlation was found between the relative thickness before training and the percentage increase in PFS $(r=-0.31$; $p<0.05)$. A significant negative correlation was also found between the relative thickness before training and the percentage increase in GMM thickness $(r=-0.37, p<0.01)$. No significant correlation was found between the relative thickness before training and the percentage increase in SoL thickness $(r=0.16, n s)$.

The percentage increase in PFS, SoL, and GMM thicknesses did not correlate significantly with age $(r=0.21$ for PFS, $n s$; $r=0.17$ for SoL, $n s ; r=0.04$ for GMM, $n s)$.

\section{Relationship between PFS and muscle thickness}

Before the training, significant positive correlations were found between PFS and GMM thickness $(r=0.45, p<0.01)$ and between PFS and the sum of thicknesses of SoL and GMM $(r=0.48, p<0.001)$. On the other hand, no significant correlation was found between PFS and SoL thickness $(r=0.27, n s)$. These correlation coefficients did not change significantly after training $(r=0.18$ for SoL, $n s ; r=0.46$ for
GMM, $p>0.01 ; r=0.42$ for the sum of the thicknesses of SoL and GMM, $p>0.01)$.

Questionnaire on the implementation of training and the subjective assessment of training effects

Almost all participants performed the training at home, in the kitchen $(67 \%)$, living room $(43 \%)$, corridor $(10 \%)$, or washroom (10\%). Main times of training were morning $(76 \%)$, daytime $(67 \%)$, and nighttime $(43 \%)$. Fifty-one percent of participants felt that the training was easy to perform. The training period was divided into three periods (1st: 1-20 days, 2nd: 21-40 days, 3rd: 41-62 days). Difficulty performing the training was reported by $49 \%$ of participants in the 1 st period, by $10 \%$ in the 2 nd period also, and by $2 \%$ (one participant) in all the periods. No participant experienced injury or other disorder due to the training. Training effects were noticed by $88 \%$ of participants; $67 \%$ felt these effects in the 2 nd period, $28 \%$ in the 1 st period, and $5 \%$ in the $3 \mathrm{rd}$ period. The training effects which many participants experienced included the following: improvement in muscle strength of the lower extremities ( $60 \%$ of participants), ease of walking and going up and down stairs (35\%), improvement in physical condition 
(35\%), avoidance of a fall when losing balance (33\%), improvement in balance $(28 \%)$, improvements in extension of the hip or knee joint (23\%), and smoother movements in daily life $(23 \%)$.

\section{Discussion}

Muscle strength training for the triceps surae has recently been proven effective in increasing PFS (percentage increase: 20-30\%) in the elderly (Ferri et al., 2003; Morse et al., 2005; Simoneau et al., 2006). The muscle load adopted in those training protocols was relatively high, at more than $50 \%$ of maximal muscle strength. In contrast, the heel-raise training in this study used a relatively low muscle load so that the exercise could be repeated 100 times. Such a low load predominantly activates slow-twitch muscle fibers (Ikai et al., 1965; Berger and Hardage, 1967; Okada, 1971). After the training, PFS showed a significant increase of $25.8 \%$, similar to that observed in the above-mentioned studies. There was no significant correlation between increase in PFS and age, suggesting that the effects of heel-raise training on PFS were applicable throughout the age group studied (60-79 years). It is suggested that the improvement in PFS was caused by increased muscle volume, which is related to increases in muscle thickness and in the number of motor units recruited (Simoneau et al., 2006; de Boer et al., 2007). The percentage increase in a cross-sectional area of the triceps surae would be approximately $20 \%$, based on the square of the mean percentage increase $(9.65 \%)$ in the thickness of SoL and GMM (12.7\% and $6.6 \%$, respectively). This percentage increase was low compared with that in PFS. However, although the present study is unable to verify the mechanism behind the improvement in PFS, we suspect that increased recruitment of motor units contributes. In addition, hypertrophy through training of the plantar flexor muscles other than the triceps surae may relate to the increase of PFS.

The percentage increase in SoL thickness was significantly greater than that in GMM thickness. This supports the hypothesis that the training effect is more remarkable in SoL on account of its high proportion of slow-twitch fibers. In addition, the relative thickness of GMM to SoL after training was significantly smaller than that before training. The difference in training-induced thickness between SoL and GMM is probably related to the two following factors: 1) the proportion of slow-twitch fibers is much higher in SoL than in GMM (Johnson et al., 1973; Trappe et al., 2001), 2) slowtwitch fibers mainly participate in muscle contraction during the heel-raise training because muscle load is low (Henneman et al., 1965; Ikai et al., 1965; Berger and Hardage, 1967; Okada, 1971). Heel-raise training may be an effective training method for slow-twitch fibers, where contraction depends on muscle blood flow (Bonde-Petersen and Robertson, 1981; Petrofsky et al., 1981).

Participants with relatively high SoL and GMM thickness and PFS before training showed smaller percentage increases in these measurements. For GMM of 12 participants who had a relatively large thickness, no increase in thickness or a slight decrease was observed. From the viewpoint of the overload principle inducing muscle hypertrophy, the load on both muscles in the heel-raise training may be comparatively low for such participants. The effects of training would therefore be less remarkable, especially for GMM, which has a relatively low proportion of slow-twitch fibers.

The percentage increase in GMM thickness significantly and negatively correlated with the relative thickness of GMM to SoL before training $(r=-0.37)$. Therefore, the relative thickness may indirectly indicate the proportion of slow-twitch muscle fibers in the triceps surae. Assuming that a low muscle load exercise is suitable for the training of slow-twitch muscle fibers, the training effect would be stronger for participants with low relative thickness before training. The significant negative correlation mentioned above may support this hypothesis.

PFS correlated significantly with GMM thickness. This may relate to the fact that twitch tension is larger in the fast-twitch fibers than in the slow-twitch fibers (Edington and Edgerton, 1976), and the maximal voluntary muscle contraction correlates strongly with the proportion of the fast-twitch muscle fibers (Aagaard and Andersen, 1998).

The results of the questionnaire showed that the main places where participants performed the training were the kitchen and living room. No participant reported injury or other disorder caused by the training. Only one participant reported that the training was difficult throughout the training period. These results suggest that the present heel-raise training program for the elderly could be performed safely and easily at home. Eighty-eight percent of the participants recognized training effects, and this mainly occurred in the 2 nd period. This period is equivalent to that noted in other studies (Ploutz et al., 1994; Young et al., 1983), and could be a guideline for the timing of training effect in the triceps surae. Subjectively, participants noted improvement of muscle strength of the leg. In addition, many participants described improvements in their balance and ability to walk. It is inferred that heel-raise training might be useful for preventing falls and the functional improvement of movements in daily life.

\section{Conclusion}

This study demonstrated that a two-month training program involving a set of 100 repetitions per day of heel-raise was effective for the elderly as a form of muscle training focused on SoL. This training can be safely and easily performed at home and in many health and rehabilitation institutions, and might prevent falls and improve quality of life.

\section{References}

Aagaard P, Andersen JL (1998) Correlation between contractile strength and myosin heavy chain isoform 
composition in human skeletal muscle. Med Sci Sports Exerc 30: 1217-1222

Berger RA, Hardage B (1967) Effect of maximum loads for each of ten repetitions on strength improvement. Res Q 38: 715-718

Bonde-Petersen F, Robertson CH Jr (1981) Blood flow in "red" and "white" calf muscles in cats during isometric and isotonic exercise. Acta Physiol Scand 12: 243-251

de Boer MD, Morse CI, Thom JM, de Haan A, Narici MV (2007) Changes in antagonist muscles' coactivation in response to strength training in older women. J Gerontol A Biol Sci Med Sci 62: 1022-1027

Edington DW, Edgerton VR (1976) The biology of physical activity. Houghton Mifflin, Boston

Ferri A, Scaglioni G, Pousson M, Capodaglio P, Van Hoecke J, Narici MV (2003) Strength and power changes of the human plantar flexors and knee extensors in response to resistance training in old age. Acta Physiol Scand 177: 69-78

Fujiwara K, Asai H, Toyama H, Kunita K, Yaguchi C, Kiyota N, Tomita H, Jacobs JV (2010) Changes in muscle thickness of gastrocnemius and soleus associated with age and sex. Aging Clin Exp Res (in press)

Fujiwara K, Ikegami H, Okada M, Koyama Y (1982) Contribution of age and muscle strength of lower limbs to steadiness and stability in standing posture. J Anthropol Soc Nippon 90: 385-400 [In Japanese with English Abstract]

Fujiwara K, Toyama H, Koyama S, Ikeda Y, Sugo K (1988) The validity and reliability of the measurement of lower leg muscle strength and its value for female university students. Ann Sci Kanazawa Univ 25: 51-60 [In Japanese with English Abstract]

Fukunaga T, Roy RR, Shellock FG, Hodgson JA, Edgerton VR (1996) Specific tension of human plantar flexors and dorsiflexors. J Appl Physiol 80: 158-165

Haxton HA (1944) Absolute muscle force in the ankle flexors of man. J Physiol 103: 267-273

Henneman E, Somjen G, Carpenter DO (1965) Excitability and inhibitability of motoneurons of different sizes. J Neurophysiol 28: 599-620

Humphreys PW, Lind AR (1963) The blood flow through active and inactive muscles of the forearm during sustained hand-grip contractions. J Physiol 166: 120-135

Ikai M, Fukunaga T (1968) Calculation of muscle strength per unit cross-sectional area of human muscle by means of ultrasonic measurement. Int Z Angew Physiol 26: 26-32

Ikai M, Ishii M, Nakamura J (1965) Muscle endurance (II). J Health Phys Ed Recrea 15: 281-287 [In Japanese]

Inman VT, Ralston HJ, Todd F (1981) Human walking. Williams \& Wilkins, Baltimore

Johnson MA, Polgar J, Weightman D, Appleton D (1973) Data on the distribution of fibre types in thirty-six human muscles. An autopsy study. J Neurol Sci 18: 111-129

Morse CI, Thom JM, Mian OS, Muirhead A, Birch KM, Narici
MV (2005) Muscle strength, volume and activation following 12-month resistance training in 70-year-old males. Eur J Appl Physiol 95: 197-204

Nadeau S, Gravel D, Arsenault AB, Bourbonnais D (1999) Plantarflexor weakness as a limiting factor of gait speed in stroke subjects and the compensating role of hip flexors. Clin Biomech (Bristol, Avon) 14: 125-135

Narici M, Cerretelli P (1998) Changes in human muscle architecture in disuse-atrophy evaluated by ultrasound imaging. J Gravit Physiol 5: 73-74

Okada M (1971) An electromyographic estimation of the relative muscular load in different human postures. J Hum Ergol 1: 75-93

Perry J (1992) Gait analysis. Slack Incorporated, Thorofare

Petrofsky JS, Phillips CA, Sawka MN, Hanpeter D, Stafford D (1981) Blood flow and metabolism during isometric contractions in cat skeletal muscle. J Appl Physiol 50: 493-502

Ploutz LL, Tesch PA, Biro RL, Dudley GA (1994) Effect of resistance training on muscle use during exercise. J Appl Physiol 76: 1675-1681

Reimers CD, Schlotter B, Eicke BM, Witt TN (1996) Calf enlargement in neuromuscular disease: a quantitative ultrasound study in 350 patients and review of the literature. J Neurol Sci 143: 46-56

Shono N, Urata H, Saltin B, Mizuno M, Harada T, Shindo M, Tanaka H (2002) Effects of low intensity aerobic training on skeletal muscle capillary and blood lipoprotein profiles. J Atheroscler Thromb 9: 78-85

Simoneau E, Martin A, Porter MM, Van Hoecke J (2006) Strength training in old age: adaptation of antagonist muscles at the ankle joint. Muscle Nerve 33: 546-555

Trappe SW, Trappe TA, Lee GA, Costill DL (2001) Calf muscle strength in humans. Int J Sports Med 22: 186-191

Wernbom M, Augustsson J, Thomeé R (2006) Effects of vascular occlusion on muscular endurance in dynamic knee extension exercise at different submaximal loads. J Strength Cond Res 20: 372-377

Young A, Stokes M, Round JM, Edwards RH (1983) The effect of high-resistance training on the strength and crosssectional area of the human quadriceps. Eur J Clin Invest 13: $411-417$

Received: June 18, 2009

Accepted: October 30, 2009

Correspondence to: Katsuo Fujiwara, Department of Human Movement and Health, Graduate School of Medical Science, Kanazawa University, 13-1 Takara-machi, Kanazawa 920-8640, Japan

Phone: $+81-76-265-2225$

Fax: +81-76-234-4219

e-mail: fujikatu@med.m.kanazawa-u.ac.jp 